\title{
Comparison of Xbox One and Steam Joystick- based Operating System User Interface using KLM- GOMS
}

\author{
Dodick Zulaimi Sudirman ${ }^{1,2}$ \\ ${ }^{1}$ Computer Science Department \\ BINUS \\ Graduate Program - Doctor of \\ Computer Science, \\ Bina Nusantara university \\ Jakarta, Indonesia 11480 \\ ${ }^{2}$ Computer Science Department \\ School of Computer Science \\ Bina Nusantara University \\ Jakarta, Indonesia 11480 \\ dodick.sudirman@binus.ac.id \\ Benfano Soewito \\ Computer Science Department \\ BINUS \\ Graduate Program - Doctor of \\ Computer Science, \\ Bina Nusantara university \\ Jakarta, Indonesia 11480 \\ bsoewito@binus.edu
}

\author{
Yaya Heryadi \\ Computer Science Department \\ BINUS \\ Graduate Program - Doctor of \\ Computer Science, \\ Bina Nusantara university \\ Jakarta, Indonesia 11480 \\ yayaheryadi@binus.edu
}

\author{
Harco Leslie Hendric Spits \\ Warnars \\ Computer Science Department \\ BINUS \\ Graduate Program - Doctor of \\ Computer Science, \\ Bina Nusantara university \\ Jakarta, Indonesia 11480 \\ spits.hendric@binus.ac.id
}

\begin{abstract}
Despite having many advances in video game control such as voice or gesture control, most video game console manufacturers still use a joystick as the main control for their console. Currently, the problem with video game manufacturers is that instead of using the joystick as the point of user interface design, they instead used a design from another device such as a computer or tv. The research will compare Xbox One and Steam OS user interface by using KLM-GOMS Model. Based on the calculation it is concluded that the overall Xbox One has a more efficient design compared to Steam.
\end{abstract}

Keywords-game controller, game console, GOMS, joystick

\section{INTRODUCTION}

Ever since the introduction of ATARI in the 1970's, video game console has emerged as an important game controller. In the past 30 years, supported by the fast- growing game industry, the console has evolved very fast. A study by Kemerer, Dunn \& Janansefat [1] concluded that the current video game can be categorized as the $8^{\text {th }}$ generation of the video game. Since the first generation of game console, video game manufacturer not only have tried to upgrade graphics or processing quality but also provided a new mechanism for the player to interact with the game.

The first game controller was an analog controller with several dial buttons used in the Magnavox Odyssey 100. It was in this era, Atari 2600 introduced a very simple joystick called CX10 as an analog stick with a single button [1]. The joystick was so popular that it was used not only for playing game but also for operating a home computer. The next generation of game console was grip form with four directions pad (d-pad) and two buttons introduced by the Nintendo Entertainment System (NES) [2]. In the following years, some evolvement of game controller still went on. For example controller for the Super Nintendo Entertainment System (SNES) [3] introduced some additional buttons. Similarly, Sony PlayStation added two analog controllers to the scheme which has successfully brought the popular first- person shooter game from personal computer to the video game console.

Innovative control scheme finally introduced on the seventh-generation video game console. Wii Remote introduced the usage of gesture console in the industry [4]. It 
was followed by Microsoft Kinect and Sony Move Controller that also uses gesture to control the game.

Despite some gesture controls were welcomed by the casual game player but the most game player still prefer regular controller [5]. According to van Beurden et al. [5], gesture control, in general, is perceived better in terms of hedonic quality and fun while performing less in pragmatic quality [5]. In the same research, the fun experience also faces a problem with the fatigue of playing the game. That is why Nintendo Switch even though still have a gesture controller they still have a Joy-con grip and Wii Pro controller. Microsoft and Sony also still use their regular controller as the main controller for their video game console.

In the past ten years, research on game controllers can be divided broadly into two directions. First, game controller hardware. The study by Ramcharitar \& Teat in 2017 [6], for example, evaluated video game controller by using Fitt's law evaluation resulted in the best experience for mouse and touchpad. Interestingly, although mouse and touchpad perform better than joystick the respondent still prefer to use a joystick [6]. Natapov, Castellucci \& MacKenzie [7] used ISO 9241-9, a standard that evaluates the non-keyboard devices, to evaluate one aspect of a video game controller with a similar result.

Another study by Költringer, Isokoski \& Grechenig [8] concluded the best possible approach on typing using a joystick. The authors showed some evidence that their design outperformed the standard qwerty keyboard. Based on the research findings, the authors argued that there were potential of video game controller enhancement both in software and user interface design.

Second, the operating system. The study by PricewaterhouseCoopers, Singapore in 2012 [9] concluded that the game console has evolved to be more than just to play the game. For example, the player can also watch movies, listen to music and surf the web [9]. For such reason, video game console manufacturer tried to come up with the interface that can cater to such needs. Microsoft uses a tile- based metro style that is similar to Windows 10 for their Xbox One video game console [11]. Sony uses its signature XMB (Cross Media Bar) in their PlayStation 4 game console that is also present in their TV and other AC devices [10]. The problem with mentioned approaches is that instead of using the joystick as the point of user interface design, video game console manufacturer simplify the experience by reusing existing design on their other device. Even valve with their Steam OS and Nintendo with their Wii did a similar mistake by focusing on their console novelty.

Despite a vast number of studies have been reported, little has been said on a study focusing on game joystick- based operating system platforms. This study aims to compare two major game joystick-based operating system platforms in term of the user interface by using GOMS (Goals, Operators, Methods and Selection) Model. The research uncover the weakness and the strength that each operating system had. The finding will be used to design a better experience for the future joy-stick based operating system.

\section{XBox ONE AND SteAm Big Picture}

In this comparison, two joystick-based operating system will be compared by using GOMS model. Xbox One is chosen because it is manufactured by Microsoft that has developed Windows which is a personal computer operating system that has the biggest user in the world. The second joystick-based operating system that is chosen is Steam Big Picture because it is the biggest PC game platform with more than $70 \%$ market share on the PC market [12].

Xbox One is the latest video game console made by Microsoft [17]. The game console is developed to handle high-performance games and other content as music and video. Xbox One uses cloud technology to constantly back up user data, help the processing of the new system and allow background update [17]. The look and feel of the operating system are similar to Windows 10 operating system with tiled metro style.

Steam Big Picture is a version of the Steam platform that's optimized for big screen [12]. It is similar to Steam OS (Operating System) which is a Linux based operating system for gaming that is the free and open source[12]. The operating system is an attempt by its developer, Valve, to move PC gaming to the living room as an alternative to video game console [12]. The user interface was built aroundtheir proprietary Steam Controller.

\section{III.RESEARCH METHOD}

\section{A. GOMS}

GOMS is the abbreviation of Goals, Operators, Methods, and Selection rules [13]. It is a method developed by Stuart K. Card, Thomas P. Moran, and Allen Newell to measure and predicting a performance of computer interface with skilled user [13]. The GOMS model assumed that tasks that user need to do are organized as Goals. The Operator is an action or tools that is executed or used by the user to achieve goals. The methods are an organized set of operators that are used to a certain goal. The selection rules specified which methods are applied under the certain condition when multiple methods are possible.

The weakness of the original GOMS is that it needs a high amount of effort to produce an accurate model. If tasks have a lot of sub-goals, methods and selection rules, GOMS become cumbersome. As a result, Stuart K. Card, Thomas P. Moran, and Allen Newell developed Keystroke Level Stroke-GOMS or KLM-GOMS that is a more streamlined version of GOMS[15].

\section{B. KLM-GOMS}

Keystroke Level Model (KLM-GOMS) purpose is to model the time for the user to accomplish a task with a prearranged method on a computer system [15]. KLMGOMS divided operators into three broad categories which are physical-motor, user's mental act (M), and system response $(\mathrm{R})$. The physical-motor operator is divided into keystrokes $(\mathrm{K})$, pointing $(\mathrm{P})$, homing $(\mathrm{H})$ and drawing $(\mathrm{D})$ [16]. The methods are arranged into a sequence of operators and the benchmark times are summed to return a time prediction for each method [15]. 
Based on David Kieras (2001) research, below are the standard benchmark time for each operator of KLM-GOMS [16]:

Table I. Operators benchmark time

\begin{tabular}{|c|c|}
\hline Operator & Time (in seconds) \\
\hline Keystroke (K) & 0.28 \\
\hline Type (T) & Total characters x K \\
\hline Press mouse button (B) & 0.1 \\
\hline Click mouse button rapidly (BB) & 0.2 \\
\hline Movement from mouse to the keyboard (H) & 0.4 \\
\hline Mental Act (M) & 1.35 \\
\hline Waiting Time (W) & $\begin{array}{c}\text { Based on system } \\
\text { response }\end{array}$ \\
\hline
\end{tabular}

David Kieras also mentions that waiting time (W) is now considered to be unnecessary because it may have the same value in all alternative design[16].

\section{Video game console goals and objectives}

As previously mentioned, video game console is not only being used for playing the game but also for consuming other media, where the player can also watch movies, listen to music and surf the web [9]. Based on PricewaterhouseCooper consumer research, below is the list of features that players usually does on their game console [9]:

1. Playing game

2. Watch movies

3. Listen to music

4. Surfing website

\section{RESUlT AND ANALYSIS}

The list of four features above will be determined as the goals for both consoles. Each step of operators in table I that needed to be executed to achieve the goal will be listed and calculated. After all, the goals are calculated, the result will be analyzed and summarized.

Button pressed in the joystick will be considered to be keystroke compared to mouse button press because unlike joystick mouse only have 2 buttons. Xbox One standard controller will be used to control both the operating systems. Since each game console has a different library of content the calculation will only go until it reach the first content (game, movie or music) on its method that can be consumed by either played, watch or listen.

\section{A. Steam Big Picture KLM - GOMS Calculation}

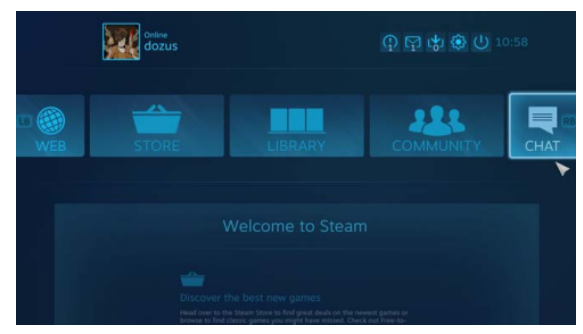

Figure I. Steam big picture main screen
Steam Big Picture is the client based platform that has the same interface and function with Steam operating system. It's main screen is depicted on Figure I.

Table II. KLM calculations for playing the game on Steam

\begin{tabular}{|c|c|c|}
\hline \multicolumn{3}{|c|}{ GOALS: Playing the game in Steam Big Picture } \\
\hline Methods & Sequence & $\begin{array}{r}\text { Duration } \\
\text { (Seconds) }\end{array}$ \\
\hline \multirow{10}{*}{$\begin{array}{l}\text { Play Gameby } \\
\text { game library }\end{array}$} & Confirm (M) & 1,35 \\
\hline & Press A $(\mathrm{K})$ & 0,28 \\
\hline & Press Down $(\mathrm{K}) \times 3$ & 0,84 \\
\hline & Press Right $(\mathrm{K})$ & 0,28 \\
\hline & Confirm (M) & 1,35 \\
\hline & Press A $(\mathrm{K})$ & 0,28 \\
\hline & Press Right $(\mathrm{K})$ & 0,28 \\
\hline & Confirm (M) & 1,35 \\
\hline & Press A $(\mathrm{K})$ & 0,28 \\
\hline & Total & 6,29 \\
\hline \multirow{10}{*}{$\begin{array}{l}\text { The play } \\
\text { recently played } \\
\text { thegame }\end{array}$} & Confirm (M) & 1,35 \\
\hline & Press A $(\mathrm{K})$ & 0,28 \\
\hline & Press Down $(\mathrm{K})$ & 0,28 \\
\hline & Press Right $(\mathrm{K})$ & 0,28 \\
\hline & Confirm (M) & 1,35 \\
\hline & Press A & 0,28 \\
\hline & Press Right & 0,28 \\
\hline & Confirm (M) & 1,35 \\
\hline & Press A & 0,28 \\
\hline & Total & 5,73 \\
\hline \multirow{10}{*}{$\begin{array}{c}\text { Play Game } \\
\text { through } \\
\text { installed games }\end{array}$} & Confirm (M) & 1,35 \\
\hline & Press A $(\mathrm{K})$ & 0,28 \\
\hline & Press Down $(\mathrm{K}) \times 2$ & 0,56 \\
\hline & Press Right $(\mathrm{K})$ & 0,28 \\
\hline & Confirm (M) & 1,35 \\
\hline & Press A $(\mathrm{K})$ & 0,28 \\
\hline & Press Right $(\mathrm{K})$ & 0,28 \\
\hline & Confirm (M) & 1,35 \\
\hline & Press A (K) & 0,28 \\
\hline & Total & 6,01 \\
\hline \multirow{6}{*}{$\begin{array}{c}\text { Play game } \\
\text { through resume } \\
\text { \& explore }\end{array}$} & Confirm (M) & 1,35 \\
\hline & Press A $(\mathrm{K})$ & 0,28 \\
\hline & Press Right $(\mathrm{K})$ & 0,28 \\
\hline & Confirm (M) & 1,35 \\
\hline & Press A & 0,28 \\
\hline & Total & 3,54 \\
\hline
\end{tabular}

There are 4 methods that can be used in Steam Big Picture to start a game. The player can access the game from the game library, recently played list, installed game list or resume \& explore the option. The KLM calculation for each method can be viewed on table II.

Table III. KLM calculations for watching a movie on Steam

\begin{tabular}{|c|c|c|}
\hline \multicolumn{2}{|c|}{ GOALS: Watching a movie in Steam Big Picture } \\
\hline \multirow{4}{*}{$\begin{array}{c}\text { Watch a movie by } \\
\text { movie library }\end{array}$} & Confirm (M) & Duration (Seconds) \\
\cline { 2 - 3 } & Press A (K) & 1,35 \\
\cline { 2 - 3 } & Press Down (K) x & 0,28 \\
\cline { 2 - 3 } & 4 & 1,12 \\
\cline { 2 - 3 } & Press Right (K) & 0,28 \\
\cline { 2 - 3 } & Confirm (M) & 1,35 \\
\cline { 2 - 3 } & Press A (K) & 0,28 \\
\cline { 2 - 3 } & Press Right (K) & 0,28 \\
\cline { 2 - 3 } & Confirm (M) & 1,35 \\
\hline
\end{tabular}


Journal of Game, Game Art and Gamification Vol. 05, No. 02, 2020

Special Issues: 2018 - International Conference of Game, Game Art and Gamification

\begin{tabular}{|c|c|c|}
\cline { 2 - 3 } & Press A (K) & 0,28 \\
\cline { 2 - 3 } & Total & $\mathbf{6 , 5 7}$ \\
\hline \multirow{4}{*}{$\begin{array}{c}\text { Watch a movie by } \\
\text { using resume } \\
\text { explore }\end{array}$} & Confirm (M) & 1,35 \\
\cline { 2 - 3 } & Press A (K) & 0,28 \\
\cline { 2 - 3 } & Press Right (K) & 0,28 \\
\cline { 2 - 3 } & Confirm (M) & 1,35 \\
\cline { 2 - 3 } & Press A & 0,28 \\
\cline { 2 - 3 } & \multicolumn{2}{|c|}{ Total } \\
\hline
\end{tabular}

Watching movie feature in Steam Big Picture can be accessed through the movie library and resume \& explore the option. The KLM calculation of watching a movie in Steam Big Picture for both method can be viewed on table III.

\begin{tabular}{|c|c|c|}
\hline \multicolumn{3}{|c|}{ GOALS: Listen to music in Steam Big Picture } \\
\hline Methods & $\begin{array}{l}\text { Sequence } \\
\end{array}$ & $\begin{array}{l}\text { Duration } \\
\text { (Seconds) }\end{array}$ \\
\hline \multirow{10}{*}{$\begin{array}{l}\text { Listen to music } \\
\text { byAlbum }\end{array}$} & Confirm (M) & 1,35 \\
\hline & Press A (K) & 0,28 \\
\hline & Press Down $(\mathrm{K}) \times 5$ & 1,4 \\
\hline & Press Right $(\mathrm{K})$ & 0,28 \\
\hline & Confirm (M) & 1,35 \\
\hline & Press A (K) & 0,28 \\
\hline & Press Right (K) & 0,28 \\
\hline & Confirm (M) & 1,35 \\
\hline & Press A $(\mathrm{K}) \times 3$ & 0,84 \\
\hline & Total & 7,41 \\
\hline \multirow{11}{*}{$\begin{array}{l}\text { Listen to music } \\
\text { byArtist }\end{array}$} & Confirm (M) & 1,35 \\
\hline & Press A (K) & 0,28 \\
\hline & Press Down $(\mathrm{K}) \times 5$ & 1,4 \\
\hline & Press Right $(\mathrm{K}) \times 2$ & 0,28 \\
\hline & Press Right (K) & 0,56 \\
\hline & Confirm (M) & 1,35 \\
\hline & Press A (K) & 0,28 \\
\hline & Press Right $(\mathrm{K}) \times 2$ & 0,56 \\
\hline & Confirm (M) & 1,35 \\
\hline & Press A $(\mathrm{K}) \times 3$ & 0,84 \\
\hline & Total & 7,97 \\
\hline \multirow{8}{*}{$\begin{array}{l}\text { Listen to music } \\
\text { fromrecently } \\
\text { added }\end{array}$} & Confirm (M) & 1,35 \\
\hline & Press A (K) & 0,28 \\
\hline & Press Down $(\mathrm{K}) \times 5$ & 1,5 \\
\hline & Press Right (K) & 0,28 \\
\hline & Press Down $(\mathrm{K})$ & 0,28 \\
\hline & Confirm (M) & 1,35 \\
\hline & Press A $(K) \times 2$ & 0,56 \\
\hline & Total & 5,5 \\
\hline \multirow{7}{*}{$\begin{array}{l}\text { Listen to music } \\
\text { fromrecently } \\
\text { played }\end{array}$} & Confirm (M) & 1,35 \\
\hline & Press A $(\mathrm{K})$ & 0,28 \\
\hline & Press Down $(\mathrm{K}) \times 5$ & 1,5 \\
\hline & Press Right $(\mathrm{K})$ & 0,28 \\
\hline & Press Down $(\mathrm{K}) \times 2$ & 0,56 \\
\hline & Confirm (M) & 1,35 \\
\hline & Press A $(\mathrm{K}) \times 2$ & 0,56 \\
\hline
\end{tabular}

In Steam Big Picture, player has 4 methods of access to listen to music. They can access it by artist, album, recently added or recently played. The KLM calculation of listening to music in Steam Big Picture for both method can be viewed on table IV.

Table V. KLM calculations for surfing the web on Steam

\begin{tabular}{|c|c|c|c|c|c|}
\hline \multicolumn{6}{|c|}{ GOALS: Surfing the web in Steam Big Picture (accessing www.bit.ly) } \\
\hline No. & Sequence & \begin{tabular}{c|} 
Dur. \\
(Sec.)
\end{tabular} & No. & Sequence & $\begin{array}{c}\text { Dur } \\
\text { (Sec.) }\end{array}$ \\
\hline 1 & Press RB (K) & 0,28 & 21 & Press Right $(\mathrm{K}) \times 5$ & 1,4 \\
\hline 2 & Press Left $(\mathrm{K})$ & 0,28 & 22 & Confirm $(\mathrm{M})$ & 1,35 \\
\hline 3 & Press Down $(\mathrm{K})$ & 0,28 & 23 & Press A (K) & 0,28 \\
\hline 4 & Confirm (M) & 1,35 & 24 & Press Left $(\mathrm{K})$ & 0,28 \\
\hline 5 & Press A $(\mathrm{K})$ & 0,28 & 25 & Press Up (K) & 0,28 \\
\hline 6 & Press Right $(\mathrm{K})$ & 0,28 & 26 & Confirm (M) & 1,35 \\
\hline 7 & Confirm (M) & 1,35 & 27 & Press A (K) & 0,28 \\
\hline 8 & Press A $(\mathrm{K}) \times 3$ & 0,84 & 28 & Press Left $(\mathrm{K}) \times 3$ & 0,84 \\
\hline 9 & Press Down $(\mathrm{K}) \times 2$ & 0,56 & 29 & Press Up (K) & 0,28 \\
\hline 10 & Press Right $(\mathrm{K})$ x 8 & 2,24 & 30 & Confirm (M) & 1,35 \\
\hline 11 & Confirm $(\mathrm{M})$ & 1,35 & 31 & Press A $(\mathrm{K})$ & 0,28 \\
\hline 12 & Press A $(\mathrm{K})$ & 0,28 & 32 & Press Down $(\mathrm{K}) \times 4$ & 1,12 \\
\hline 13 & Press Right $(\mathrm{K}) \times 2$ & 0,56 & 33 & Press Right $(\mathrm{K})$ & 0,28 \\
\hline 14 & Press Up (K) x 2 & 0,56 & 34 & Confirm (M) & 1,35 \\
\hline 15 & Confirm (M) & 1,35 & 35 & Press A $(\mathrm{K})$ & 0,28 \\
\hline 16 & Press A (K) & 0,28 & & Total & 26,15 \\
\hline 17 & Press Left $(\mathrm{K}) \times 3$ & 0,84 & & & \\
\hline 18 & Confirm (M) & 1,35 & & & \\
\hline 19 & Press A $(\mathrm{K})$ & 0,28 & & & \\
\hline 20 & Press Down $(\mathrm{K}) \times 2$ & 0,56 & & & \\
\hline
\end{tabular}

Steam Big Picture has a built-in web browser that can be used by the player to surf the web. Table V shows the KLM calculation of browsing the web for Steam Big Picture.

All of the KLM calculations for Steam Big Picture are summarized in Table VI. At a glance, we can see that the method are varied with only some that are similar between each content.

Table VI. Summary of KLM calculations for Steam Big Picture

\begin{tabular}{|c|c|c|}
\hline Goals & Methods & Total $\begin{array}{c}\text { Duration } \\
\text { (Seconds) }\end{array}$ \\
\hline \multirow{4}{*}{ Playing game } & with all game library & 6,29 \\
\cline { 2 - 3 } & By recently played game & 5,73 \\
\cline { 2 - 3 } & By installed games & 6,01 \\
\cline { 2 - 3 } & Resume \& explore option & 3,54 \\
\hline Watch movie & with all movie library & 6,57 \\
\cline { 2 - 3 } & Resume \& explore option & 3,54 \\
\hline \multirow{3}{*}{ Listen tomusic } & By Album & 7,41 \\
\cline { 2 - 3 } & By artist & 7,97 \\
\cline { 2 - 3 } & By recently added & 5,5 \\
\cline { 2 - 3 } & By recently played & 5,8 \\
\hline Surf the web & Direct access & 26,15 \\
\hline
\end{tabular}

\section{B. Xbox One KLM-GOMS Calculation}

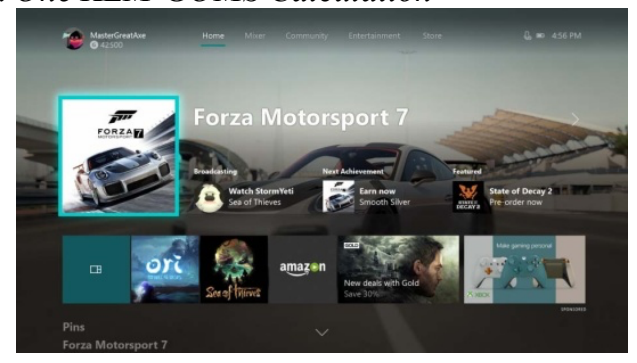

Figure II. Xbox one main screen [19] 
Journal of Game, Game Art and Gamification Vol. 05, No. 02, 2020 Special Issues: 2018 - International Conference of Game, Game Art and Gamification

Xbox One made uses Microsoft Windows 10 tile design as the basis of its operating system. It's main screen is depicted on Figure II.

Table VII. KLM calculations for playing the game on Xbox One

\begin{tabular}{|c|c|c|}
\hline \multicolumn{3}{|c|}{ GOALS: Playing the game in Xbox One } \\
\hline Methods & Sequence & $\begin{array}{l}\text { Duration } \\
\text { (Seconds) }\end{array}$ \\
\hline \multirow{3}{*}{$\begin{array}{l}\text { Play game } \\
\text { through last } \\
\text { games/apps }\end{array}$} & Confirm (M) & 1,35 \\
\hline & Press A $(\mathrm{K})$ & 0,28 \\
\hline & Total & 1,63 \\
\hline \multirow{5}{*}{$\begin{array}{c}\text { Play game } \\
\text { through most } \\
\text { recent } \\
\text { games/apps } \\
\text { list }\end{array}$} & Press Down $(\mathrm{K})$ & 0,28 \\
\hline & Press Right $(\mathrm{K})$ & 0,28 \\
\hline & Confirm (M) & 1,35 \\
\hline & Press A $(\mathrm{K})$ & 0,28 \\
\hline & Total & 2,19 \\
\hline \multirow{9}{*}{$\begin{array}{l}\text { Play game } \\
\text { using gameand } \\
\text { apps library }\end{array}$} & Press Down $(\mathrm{K})$ & 0,28 \\
\hline & Confirm (M) & 1,35 \\
\hline & Press A $(\mathrm{K})$ & 0,28 \\
\hline & Confirm (M) & 1,35 \\
\hline & Press A $(\mathrm{K})$ & 0,28 \\
\hline & Press Down $(\mathrm{K})$ & 0,28 \\
\hline & Confirm (M) & 1,35 \\
\hline & Press A $(\mathrm{K})$ & 0,28 \\
\hline & Total & 5,45 \\
\hline
\end{tabular}

There are 3 approaches that can be used in Xbox One to start a game. The player can access the game from the game $\&$ apps library, accessing last games/apps option or most recent games/apps list. The KLM calculation is listed on table VII.

Table VIII. KLM calculations for watching movies on Xbox One

\begin{tabular}{|c|c|c|}
\hline \multicolumn{3}{|c|}{ GOALS: Watch movies on Xbox One } \\
\hline Methods & Sequence & Duration (Seconds) \\
\hline \multirow{3}{*}{$\begin{array}{l}\text { Watch movie } \\
\text { through last } \\
\text { games or apps }\end{array}$} & Confirm (M) & 1,35 \\
\hline & Press A (K) & 0,28 \\
\hline & Total & 1,63 \\
\hline \multirow{5}{*}{$\begin{array}{l}\text { Play game } \\
\text { through most } \\
\text { recent games or } \\
\text { apps list }\end{array}$} & Press Down $(\mathrm{K})$ & 0,28 \\
\hline & Press Right (K) & 0,28 \\
\hline & Confirm (M) & 1,35 \\
\hline & Press A (K) & 0,28 \\
\hline & Total & 2,19 \\
\hline \multirow{16}{*}{$\begin{array}{l}\text { Watch movie } \\
\text { using game and } \\
\text { apps library }\end{array}$} & Press Down $(\mathrm{K})$ & 0,28 \\
\hline & Confirm (M) & 1,35 \\
\hline & Press A (K) & 0,28 \\
\hline & Press Down $(\mathrm{K})$ & 0,28 \\
\hline & Confirm (M) & 1,35 \\
\hline & Press A (K) & 0,28 \\
\hline & Press Down $(\mathrm{K}) \times 2$ & 0,56 \\
\hline & Press Right (K) x 3 & 0,84 \\
\hline & Press Down $(\mathrm{K})$ & 0,28 \\
\hline & Confirm (M) & 1,35 \\
\hline & Press A (K) & 0,28 \\
\hline & Press Right $(\mathrm{K})$ & 0,28 \\
\hline & Press Down $(\mathrm{K})$ & 0,28 \\
\hline & Confirm (M) & 1,35 \\
\hline & Press A (K) & 0,28 \\
\hline & Total & 9,32 \\
\hline
\end{tabular}

Watching a movie in Xbox one can be accessed with 3 methods. The player can watch a movie by going through movie library, accessing last games/apps option or most recent games/apps list. The KLM calculation of watching a movie in Xbox One for each method can be viewed in table VIII.
In Xbox One, player has 3 methods of access to listen to music. They can access it from the game \& apps library, accessing last games/apps option or most recent games/apps list. The KLM calculation of listening to music in Xbox One for each method can be viewed in table IX.

Table IX. KLM calculations for listening to music on Xbox One

\begin{tabular}{|c|c|c|}
\hline \multicolumn{3}{|c|}{ GOALS: Listen to music in Xbox One } \\
\hline Methods & Sequence & $\begin{array}{r}\text { Duration } \\
\text { (Seconds) }\end{array}$ \\
\hline \multirow{5}{*}{$\begin{array}{l}\text { Listen to } \\
\text { music } \\
\text { through last } \\
\text { games or }\end{array}$} & Confirm (M) & 1,35 \\
\hline & Press A $(\mathrm{K})$ & 0,28 \\
\hline & Confirm (M) & 1,35 \\
\hline & Press A (K) & 0,28 \\
\hline & $\begin{array}{r}\text { Total } \\
\end{array}$ & 3,26 \\
\hline \multirow{5}{*}{$\begin{array}{l}\text { Listen to } \\
\text { music } \\
\text { through most } \\
\text { recent games }\end{array}$} & Press Down $(\mathrm{K})$ & 0,28 \\
\hline & Press Right $(\mathrm{K})$ & 0,28 \\
\hline & Confirm (M) & 1,35 \\
\hline & Press A (K) & 0,28 \\
\hline & Confirm (M) & 1,35 \\
\hline \multirow{2}{*}{ or apps list } & Press A $(\mathrm{K})$ & 0,28 \\
\hline & Total & 3,82 \\
\hline \multirow{13}{*}{$\begin{array}{l}\text { Listen to } \\
\text { music using } \\
\text { game and } \\
\text { apps library }\end{array}$} & Press Down $(\mathrm{K})$ & 0,28 \\
\hline & Confirm (M) & 1,35 \\
\hline & Press A (K) & 0,28 \\
\hline & Press Down $(\mathrm{K})$ & 0,28 \\
\hline & Confirm (M) & 1,35 \\
\hline & Press A (K) & 0,28 \\
\hline & Press Down $(\mathrm{K}) \times 2$ & 0,56 \\
\hline & Press Right (K) x 2 & 0,84 \\
\hline & Confirm (M) & 1,35 \\
\hline & Press A $(\mathrm{K})$ & 0,28 \\
\hline & Confirm (M) & 1,35 \\
\hline & Press A (K) & 0,28 \\
\hline & Total & 8,48 \\
\hline
\end{tabular}

Xbox One uses Microsoft Edge as their built-in web browser that can be used by the player to surf the web. Table X shows the KLM calculation of surfing the web for Xbox One.

Table XI. KLM calculations for surfing the web on Xbox One

\begin{tabular}{|c|c|c|c|c|c|}
\hline \multicolumn{6}{|c|}{ GOALS: Surfing the web in Xbox One (accessing www.bit.ly) } \\
\hline No. & Sequence & $\begin{array}{c}\text { Dur } \\
\text { (Sec.) }\end{array}$ & No. & Sequence & $\begin{array}{c}\text { Dur } \\
\text { (Sec.) }\end{array}$ \\
\hline 1 & Press Down (K) & 0,28 & 22 & Press Left (K) x 3 & 0,84 \\
\hline 2 & Confirm (M) & 1,35 & 23 & Confirm (M) & 1,35 \\
\hline 3 & Press A (K) & 0,28 & 24 & Press A (K) & 0,28 \\
\hline 4 & Press Down (K) & 0,28 & 25 & Press Down (K) x 2 & 0,56 \\
\hline 5 & Confirm (M) & 1,35 & 26 & Press Right (K) x 5 & 1,4 \\
\hline 6 & Press A (K) & 0,28 & 27 & Confirm (M) & 1,35 \\
\hline 7 & Press Right (K) & 0,28 & 28 & Press A (K) & 0,28 \\
\hline 8 & Press Down (K) & 0,28 & 29 & Press Left (K) & 0,28 \\
\hline 9 & Press Y (K) & 0,28 & 30 & Press Up (K) & 0,28 \\
\hline 10 & Press A (K) & 0,28 & 31 & Confirm (M) & 1,35 \\
\hline 11 & Press Left (K) x 4 & 1,12 & 32 & Press A (K) & 0,28 \\
\hline 12 & Confirm (M) & 1,35 & 33 & Press Left (K) x 3 & 0,84 \\
\hline \multicolumn{7}{|c}{} & \multicolumn{3}{|}{}
\end{tabular}




\begin{tabular}{|c|c|c|c|c|c|}
\hline 13 & Press A (K) $\times 3$ & 0,84 & 34 & Press Up (K) & 0,28 \\
\hline 14 & Press Down (K) $\times 2$ & 0,56 & 35 & Confirm (M) & 1,35 \\
\hline 15 & Press Right (K) 8 & 2,24 & 36 & Press A (K) & 0,28 \\
\hline 16 & Confirm (M) & 1,35 & 37 & Press Down (K) 33 & 0,84 \\
\hline 17 & Press A (K) & 0,28 & 38 & Press Right (K) $\times 2$ & 0,28 \\
\hline 18 & Press Right (K) 2 & 0,56 & 39 & Confirm (M) & 1,35 \\
\hline 19 & Press Up (K) $\times 2$ & 0,56 & 40 & Press A (K) & 0,28 \\
\hline 20 & Confirm (M) & 1,35 & & \multicolumn{5}{|c}{ Total } & $\mathbf{2 9 , 1 8}$ \\
\hline 21 & Press A (K) & 0,28 & \multicolumn{4}{|l}{} \\
\hline
\end{tabular}

All of the KLM calculations for Xbox One are summarized in Table XI. By design, Microsoft used similar steps and methods in order to access all type of contents. Microsoft also provide a shortcut method that will help player to access the content that they previously accessed faster compared to the conventional methods. Surfing the web is the only activity that has only one methods and different compared to other methods.

Table XI. Summary of KLM calculations for Xbox One

\begin{tabular}{|c|c|c|}
\hline \multirow{2}{*}{ Goals } & Methods & $\begin{array}{c}\text { Duration } \\
\text { (Seconds) }\end{array}$ \\
\hline \multirow{3}{*}{ Playing game } & Through last game/apps & 1,63 \\
\cline { 2 - 3 } & Through most recent games/apps list & 2,19 \\
\cline { 2 - 3 } & Using game and apps library & 6,01 \\
\hline \multirow{3}{*}{ Watch movie } & Through last game/apps & 1,63 \\
\cline { 2 - 3 } & Through most recent games/apps list & 2,19 \\
\cline { 2 - 3 } & Using game and apps library & 9,32 \\
\hline \multirow{2}{*}{$\begin{array}{c}\text { Listen to } \\
\text { music }\end{array}$} & Through last game/apps & 3,26 \\
\cline { 2 - 3 } & Through most recent games/apps list & 3,82 \\
\cline { 2 - 3 } & Using game and apps library & 8,8 \\
\hline Surf the web & Direct access & 29,18 \\
\hline
\end{tabular}

\section{Analysis}

The comparison of both KLM calculations is presented in Table XII. Before we look at the number of KLM calculations, we will analyze the number of method for both game operating system. It shows Xbox One has a consistent number of methods that can be used to access content (game, movie, and music) compared to Steam. Not only Xbox One has a consistent number of methods, it also has a similar sequence.

Table XII. KLM calculations comparison for Xbox One and Steam

\begin{tabular}{|c|c|c|c|r|c|}
\hline \multicolumn{2}{|c|}{ Goals } & $\begin{array}{r}\text { Playing } \\
\text { Game }\end{array}$ & $\begin{array}{r}\text { Watch } \\
\text { movie }\end{array}$ & $\begin{array}{c}\text { Listen to } \\
\text { music }\end{array}$ & $\begin{array}{c}\text { Surf the } \\
\text { web }\end{array}$ \\
\hline $\begin{array}{c}\text { Total } \\
\text { methods }\end{array}$ & Steam & 4 & 2 & 4 & 1 \\
\cline { 2 - 6 } & Xbox & 3 & 3 & 3 & 1 \\
\hline Average & Steam & 5,39 & 5,06 & 6,67 & 26,15 \\
\cline { 2 - 6 } Duration & Xbox & 3,28 & 4,38 & 5,29 & 29,18 \\
\hline $\begin{array}{c}\text { Least } \\
\text { Duration }\end{array}$ & Steam & 3,54 & 3,54 & 5,5 & 26,15 \\
\cline { 2 - 6 } & Xbox & 1,63 & 1,63 & 3,26 & 29,18 \\
\hline Most & Steam & 6,29 & 6,57 & 7,97 & 26,15 \\
\cline { 2 - 6 } Duration & Xbox & 6,01 & 9,32 & 8,8 & 29,18 \\
\hline
\end{tabular}

On average, Xbox One outperform Steam by having the least time on accessing content. Xbox One also has the fastest duration of accessing content compared to Steam. It because they have a method by accessing content through last game/apps require by simply pressing a button after the device is turned on. This shortcut method will help a player that wanted to enjoy the same content consecutively each time they use Xbox One.

The problem with Xbox One shows on the most duration data. Before player able to use the fast method through last game/apps option, the player needs to go through the long sequence to access the content that they wanted. Similar problem with Xbox One also arises when player needs to surf the web. It took longer for a player to browse the web compared to Steam.

\section{CONCLUSIONS}

Based on the KLM-GOMS calculation, Xbox One user interface has proven to be more effective compared to Steam. Xbox One also uses similar methods to access most of their content, it will help to reduce the learning curve of using the user interface. Other video game console operating system should incorporate Xbox One shortcut methods when designing their user interface. Steam also has a similar shortcut method but the access is not as fast and as efficient as the one that Xbox One uses. Despite having the most efficient operating system in overall, the Xbox One still lack in performance compared to Steam while accessing specific content without shortcut methods.

In the future, there is more video game console user interface that needs to be assessed and compared. Another note on KLM-GOMS is that since it focuses on computer application, maybe new methods are needed for joystick- based application including operating system and game.

\section{REFERENCES}

[1] C.F. Kemerer, B.K. Dunn, and B. Janansefat, "Winners-Take-Some dynamics in digital platform markets: a reexamination of the video game console wars," unpublished

[2] B. Merdenyan, and H. Petrie, "User teviews of gamepad controllers: a source of user requirements and user experience" in Proceedings of the 2015 Annual Symposium on Computer-Human Interaction in Play, 2015.

[3] N. Bhonker, S. Rozenberg, and I. Hubara, "Playing snes in the retro learning environment," in 5th International Conference on Learning Representations, 2017.

[4] T. Schlomer, B. Poppinga, N. Henze, \& S. Boll, "Gesture recognition with a wii controller", in Proceedings of the $2^{\text {nd }}$ International Conference on Tangible and Embedded Interaction, 2008.

[5] M.H.P.H. van Beurden, W.A. Ijsselsteijn, \& Y.A.W. de Kort, (2012) "User experience of gesture based i: a comparison with traditional interaction methods on pragmatic and hedonic qualities," in Efthimiou E., Kouroupetroglou G., Fotinea SE. (eds) Gesture and Sign Language in Human-Computer Interaction and Embodied Communication, 2012.

[6] A. Ramcharitar, and R.J. Teat, "a Fitts' law evaluation of video game controllers: thumbstick, touchpad and gyrosensor," in Proceedings of the 2017 CHI Conference Extended Abstracts on Human Factors in Computing Systems, 2017.

[7] D. Natapov, S.J. Castellucci, and I.S. MacKenzie, "ISO 9241-9 evaluation of video game controllers," in Proceedings of Graphics Interface, 2009.

[8] T. Költringer, P. Isokoski, and T. Grechenig, "TwoStick: writing witha game controller," in Proceedings of Graphics Interface, 2007.

[9] PricewaterhouseCooper Singapore. (2012). "The evolution of video gaming and content consumption," PwC Video Gaming and Content Consumer Intelligence, 2012. Accessed on: Jun. 5, 2018. [online] 
Journal of Game, Game Art and Gamification Vol. 05, No. 02, 2020 Special Issues: 2018 - International Conference of Game, Game Art and Gamification

Available: https://www.pwc.com/sg/en/tice/assets/ticenews201206/ evolutionvideogame201206.pdf

[10] DS. Lee, "The effect of visualizing the flow of multimedia content among and inside devices," in Journal of Applied Ergonomics Vol $40,2008$.

[11] P. Tassi,"Why can't microsoft get its xbox one dashboard right?," Forbes.com, 2017. Accessed on: April 22, 2018, Available: https://www.forbes.com/sites/insertcoin/2017/08/09/whycant- microsoft-get-its-xbox-one-dashboard-right/

[12] J. Rowe, "Valve's Contribution to the Gaming World." in J. Sharpe, and R. Self, Computers for Everyone. $1^{\text {st }}$ Edition, University of Derby, 2014.

[13] B.E. John, and D.E. Kieras, "The GOMS family of user interface analysis techniques: comparison and contrast," in ACM Transactions on Computer-Human Interaction, Vol. 3, No. 4, 1996.

[14] B.E. John, and D.E. Kieras, (1996). "Using GOMS for user interface design and evaluation: which technique?," in ACM Transactions on
Computer-Human Interaction, Vol. 3, No. 4, 1996.

[15] S.K. Card, T.P., Moran, and A. Newell, "The Keystroke-Level model for user performance time with interactive systems," in Communications of the ACM Magazine, 1980.

[16] D. Kieras, "Using the keystroke-level model to estimate execution times," unpublished, 2001.

[17] M. George, "Digital distribution unstoppable business model or gratuitous content for the masses?," in J. Sharpe, and R. Self, Computers for Everyone. $1^{\text {st }}$ Edition, University of Derby, 2014.

[18] H. Pangestu, Penerapan metode GOMS untuk evaluasi perangkat lunak pemodelan visual berbasis open source," in ComTech Journal Vol 3, No 1, 2012.

[19] M. Ybarra, "New fall update for xbox one available for everyone today," Microsoft xbox official site, 2017. Accessed on: Jul. 22, 2018,Available: https://news.xbox.com/enus/2017/10/16/fall-update- xbox-one-2017// 